\title{
Investigating the Impact of Annotation Interfaces on Player Performance in Distributed Multiplayer Games
}

\author{
Sultan A. Alharthi, Ruth C. Torres, 1 Ahmed S. Khalaf, \\ Zachary O. Toups, Igor Dolgov? 2 Lennart E. Nacke ${ }^{3}$ \\ ${ }^{1}$ Play \& Interactive Experiences for Learning Lab, New Mexico State University, Las Cruces, NM, USA \\ ${ }^{2}$ Perception, Action, \& Cognition in Mediated Artificial \& Natural Environments Lab, \\ New Mexico State University, Las Cruces, NM, USA \\ ${ }^{3}$ HCI Games Group, Games Institute, University of Waterloo, Waterloo, ON, Canada \\ \{salharth, rutorres, khalaf\}@nmsu.edu,z@cs.nmsu.edu,id@nmsu.edu, lennart.nacke@acm.org
}

\begin{abstract}
In distributed multiplayer games, it can be difficult to communicate strategic information for planning game moves and player interactions. Often, players spend extra time communicating, reducing their engagement in the game. Visual annotations in game maps and in the gameworld can address this problem and result in more efficient player communication. We studied the impact of real-time feedback on planning annotations, specifically two different annotation types, in a custom-built, third-person, multiplayer game and analyzed their effects on player performance, experience, workload, and annotation use. We found that annotations helped engage players in collaborative planning, which reduced frustration, and shortened goal completion times. Based on these findings, we discuss how annotating in virtual game spaces enables collaborative planning and improves team performance.
\end{abstract}

\section{ACM Classification Keywords}

H.5.3. Group and Organization Interfaces: CSCW

\section{Author Keywords}

Planning; sensemaking; collaboration; coordination; annotation; game design; distributed multiplayer games

\section{INTRODUCTION}

Communication is paramount for cooperation in online games, but can be difficult to achieve unless a game's interface integrates communication tools. While communication can emerge ad hoc, it often takes extra time and impedes player engagement with games. However, user interface tools can help players plan and collaborate more effectively in a game. To support team communication and planning in distributed games, players currently use some combination of communication channels: voice, text, and cooperative communication mechanics (CCMs) - mechanics that enable players' collaboration directly through gameplay [49]. CCMs help players

Permission to make digital or hard copies of all or part of this work for personal or classroom use is granted without fee provided that copies are not made or distributed for profit or commercial advantage and that copies bear this notice and the full citation on the first page. Copyrights for components of this work owned by others than the author(s) must be honored. Abstracting with credit is permitted. To copy otherwise, or republish, to post on servers or to redistribute to lists, requires prior specific permission and/or a fee. Request permissions from Permissions@acm.org.

CHI 2018, April 21-26, 2018, Montreal, QC, Canada

(C)2018 Copyright is held by the owner/author(s). Publication rights licensed to ACM. ACM 978-1-4503-5620-6/18/04 \$ \$15.00

DOI: https : //doi .org/10 . 1145/3173574. 3173888 share information, create plans, and coordinate moves. They range from avatar gestures and map pings to freehand annotations $[34,49,59]$. Annotations are freely drawn lines and shapes on top of the gameworld $[39,59]$ that allow players not only to plan new strategies efficiently, but also to review how effective their current collaboration strategies are (e.g., annotations are used by commentators and streamers [24] to explain plans and strategy to viewers in Dota 2 matches $[54,59]$ ). Overall, collaborative planning is a crucial part of distributed multiplayer games and facilitates the coordination of players.

Collaboration among team members requires that they have a shared understanding of objectives and plans and that they communicate effectively [22]. In co-located environments, collaboration and sensemaking can be established easily $[11,27,56]$; however, this is challenging in distributed virtual environments (e.g., games) [23]. For example, existing collaborative digital games provide teams with only limited interfaces and mechanics that facilitate collaborative planning [48]. While prior literature has recognized the importance of CCMs to support teamwork in games $[34,49,52,59]$, no research has yet examined how annotation tools can facilitate collaborative planning in distributed multiplayer games, how annotations impact player performance, and how player experience and workload change when these tools are available. Based on these emerging research questions and our study of related work, the hypotheses driving our study are:

H1a: using annotations shortens game goal completion times compared to not using annotations;

H1b: annotations shorten goal completion times more when visible in the map and gameworld (map-plus-gameworld) than when visible in the map alone; and

H2: player dyads annotate more when annotations are visible in the map-plus-gameworld than when they are solely visible on the map.

To evaluate CCMs for annotation and test our hypotheses, we developed the Team Coordination \& Planning Game (TeCP), a third-person-perspective cooperative puzzle game for two players [4]. Players can plan actions in advance through the use of annotation interfaces, move and carry objects, and maneuver their character through a number of obstacles to 
escape. Players communicate with each other using text chat; depending on the study condition, they may also have access to annotations on the map-only or annotations on the mapplus-gameworld. The design of the game was informed by our prior research on disaster response planning [48].

Twelve dyads (i.e., pairs of people) each played the game in the following conditions: (1) no annotations, (2) map-only annotations, and (3) map-plus-gameworld annotations. We recorded and analyzed how annotation interfaces impact player performance, workload, and experience playing the game. Our findings indicate that annotation interfaces reduced frustration, quickened communication, and helped players engage in collaborative planning, which improved their actual and perceived performance in the game.

We also found that players annotated significantly more in the map-plus-gameworld condition as compared to when they could only annotate the map. Nearly all players $(91.7 \%)$ reported finding annotations useful, yet, at the same time, we observe that such annotations are almost non-existent in current games. Based on our observations of the gameplay, we present five distinct use cases for annotations in collaborative games: (1) real-time way-guiding, (2) marking locations and objects, (3) handwriting messages, (4) expressing emotions, and (5) spamming.

The research presented in this paper makes two contributions: First, we contribute five use cases for in-game annotations discussed above. Second, we present their implications for the design of collaborative games. We discuss how annotation interfaces can support collaborative planning, how they can reduce the workload of players, why their use increases when they are visible in both the gameworld and map, and finally we show how prior game experience affect annotation use.

These annotation interfaces can be useful beyond games for virtual training, such as disaster response and combat. Our long-term research plan is to investigate how using these types of interfaces in training simulations could help responders engage in collaborative planning activities. Besides the physicalworld implications of planning mechanics, they could aid in the training of e-sports athletes and can facilitate team-based training for digital games.

\section{RELATED WORK}

In this section, we synthesize prior research on team coordination, collaborative planning and sensemaking, game mechanics, and cooperative communication mechanics.

\section{Team Coordination}

A team is defined as a group of two or more people, who are assigned different roles and collaborate to achieve a shared goal [42]. In teamwork, performance can be maximized when teams organize their activities, synchronize their effort, and maintain shared situation awareness [2,23]. Situation awareness refers to the ability of an individual to understand the state of the environment and predict future states $[13,14]$. Awareness is critical in distributed teams where team members must inform one another, both explicitly and implicitly, of personal status $[23,50]$. Shared mental models and situation awareness support teams in working together efficiently $[18,43]$.
A mental model is a way in which individuals maintain and manipulate a representation of the functioning of an object or process in their heads [31]. A model is a form of internal simulation based on experience, enabling high-level problem solving and prediction. When mental models are congruent among teammates, enabling them to simulate the world in similar ways, they are shared. Shared mental models help teams work together more efficiently, enabling implicit and nonverbal communication through the use of artifacts, reference signs, and deep understanding of team activities [5, 36, 50]. Annotation interfaces are one method for sharing a mental model quickly.

\section{Collaborative Planning and Sensemaking}

In cognitive science, plans are defined as a series of anticipated actions designed to achieve a preconceived objective [46]. Planning activities are undertaken by individuals to make decisions and synchronize effort. During any planning process, information is gathered and analyzed from different sources to establish a strategy that will lead to accomplishing a goal. Collaborative planning is undertaken by teams to develop a set of actions that can lead to solving a problem or achieving a shared goal. Shared mental models and sensemaking are important for the success of collaborative planning.

Sensemaking is an individual or social process performed to understand a situation and make decisions [58]. Such work involves identifying, searching, filtering, sharing, and synthesizing information from diverse sources to develop shared mental models and situation awareness, leading to successful collaborative planning $[2,58]$.

Current games that use planning as a gameplay activity, employ time-critical gameplay challenges (e.g., Due Process [21], Dota 2 [54]), meaning players have limited time to communicate and create strategies because of time pressure imposed by the game. Based on our prior research on disaster-responseplanning [48], we see an opportunity to develop game mechanics and interfaces that engage players in collaborative planning activities in games.

\section{Game Design}

Salen and Zimmerman [44] characterize games as interconnected systems of rules and play. Rules are the boundaries that constrain player action, the logical and mathematical structures of the game. Play is the freedom to make decisions within the rules. Game mechanics are the choices, constructed by the game designer, that a player makes, resulting in an observable outcome $[1,44]$. Mechanics that are repeatedly invoked, and that affect the underlying subsystems of the game in important ways, are the core mechanics.

Jørgensen [32] defines "gameworld" as "an information space and an ecological environment designed with certain gameplay activities in mind" [32, p23-24]. They are thus virtual spaces, inhabited by avatars, that serve as human-computer interfaces to a game system. The gameworld enforces the rules of the game, providing virtual and formal boundaries to the game. In the present research, we differentiate the gameworld interface from the map interface. 
Game design patterns support the creation of games with a vocabulary that allows us to analyze them [7]. Patterns describe replicable combinations of rules and game mechanics that serve a specific purpose in a design. We leveraged previously developed patterns to inform the design of $T e C P$.

\section{Cooperative Communication Mechanics}

Communication channels, verbal and non-verbal, are important aspects of distributed multiplayer games, enabling players to coordinate action $[9,47,57]$. Cooperative communication mechanics (CCMs) are game mechanics that support communication and enable shared references in gameworlds [49]. Toups et al. [49] identified and classified the types of CCMs that enable cooperative play and planning in games.

Annotations, visual or textual, are user interface elements that can be overlaid on top of images [41], videos [17], or gameworlds [59]. These annotations can be used to convey different information. In games, free-hand annotations are freely drawn visual lines and shapes on top of the gameworld $[39,59]$ that allow players to plan strategies and mark locations. These annotation and drawing systems serve as CCMs $[15,49]$.

Pings, visual or auditory signals that can be placed in the gameworld or minimap to focus a player's attention, are a common way to point out parts of the gameworld $[34,49,59]$. Vaddi et al. [52] studied how avatar gestures and pings in the game Portal 2 impact player performance. The authors found that CCMs were critical to coordinating actions in the game. When CCMs were combined with verbal communication, players' performances improved significantly.

Although a number of games include CCMs, their usage during gameplay can be affected by how they were designed as well as how they are provided to players in games. Wuertz et al. [59] investigated the reasons behind usage of pings and annotations in the popular multiplayer online battle arena (MOBA) game Dota 2. The authors show that players use the tools for a number of reasons such as planning, issuing warnings, pointing out resources, alerting other players to enemy contact, requesting help, and venting frustration. Comparing pings and annotations, the authors conclude that pings are used more than annotations in Dota 2. They argue that the low usage of annotations is due to the time and effort they take to create and that the annotations only appear on the minimap, which makes them less visible. The authors suggest that the ability to create and view annotations directly in the gameworld might increase their usage.

Meanwhile, Leavitt et al. [34] investigated how non-verbal cues have the potential to improve players' performances in the (MOBA) game League of Legends [40]. The study found that the number of pings and players' performance differed significantly based on players' roles and activity in both team and individual tasks. The authors argue that providing players with a variety of tools for quick, concise communication is important in time-critical games. Although the study provides insights into non-verbal communication in games, the authors focused solely on pings and did not research annotations.

The related work raises questions about how players' planning activities take place in distributed multiplayer games and how

\begin{tabular}{ll} 
Variable & \multicolumn{1}{c}{ Description } \\
Annotation tools (IV) & $\begin{array}{l}\text { Annotations that are visible in the map- } \\
\text { plus-gameworld; annotations that are } \\
\text { visible in the map-only; no annotations } \\
\text { Time spent on meeting all goals in sec- } \\
\text { onds }\end{array}$ \\
Level completion time (DV) & $\begin{array}{l}\text { Number of times the dyads used annota- } \\
\text { tions }\end{array}$ \\
Annotations usage (DV) & $\begin{array}{l}\text { GEQ is used to assess various aspects of } \\
\text { game experience }\end{array}$ \\
Game experience (DV) & $\begin{array}{l}\text { NASA TLX is used to assess workload } \\
\text { in each condition }\end{array}$
\end{tabular}

Table 1. Description of the variables in this within-subject study.

they impact performance, (cognitive) workload, experience, and usage.

\section{METHODOLOGY}

In this section, we discuss the methods used to evaluate our hypotheses. We provide a detailed description of the process of recruiting participants, our hypotheses and experimental design, measures used, the game design, and study protocol.

\section{Participants}

To recruit participants, emails and fliers were distributed to invite participants from the University of Waterloo, Canada and local community members to participate in the formal experiment. A CAD 10 gift card compensation from Tim Hortons (local coffee store) was given to each participant.

Data collection occurred over a three-week period in the summer of 2017. 12 dyads ( $N=24,6$ female, 18 male) were recruited for the study; seven dyads were all male, one all female, and 4 mixed. The average age of the participants was 24 years $(S D=4.5, N=24)$. Regarding education background: over $66 \%$ of participants were presently pursuing or completed a Bachelors or Masters degree.

\section{Hypotheses and Experimental Design}

The experiment was conducted with one condition: annotation tools. Game levels were specially designed collaborative tasks that players needed to complete to finish each level. Annotation interfaces were counterbalanced among participants, but game levels were always run in the same order.

\section{Statistical Design}

For the current study, we used a within-subjects design with a single independent variable (IV): annotation condition, with three levels representing the type of annotation interfaces available to players: annotations that are visible on the map-plusgameworld; annotations that are visible on the map-only; and no annotations (Table 1). The dependent variables (DVs) were time taken to complete the assigned goals (with a maximum of 15 minutes), annotation use counts, and scores on the game experience and workload measures (see Study Protocol). Our primary hypothesis was:

H1a: Using annotations, regardless of annotation type, results in quicker level/goal completion times than when not using annotations. 
We expected that the dyads would perform best when annotations are visible in the map and gameworld, less well when annotations are visible only on the map, and worst with no annotations. Thus, our secondary hypotheses was:

H1b: Using annotations that are visible in the gameworld and in the map leads to quicker level/goal completion times than when annotating the map solely.

Additionally, we expected that the usage of the different annotation tools would vary based on the visibility of these annotations in the game. Thus, we hypothesized that:

H2: Players use more annotations in the map-plusgameworld condition than in the map-only condition.

We expected that annotations in collaborative games, of various types, would impact player experience and workload as compared to gameplay where annotation tools are absent. Since these investigations are exploratory, we did not put forth directional hypotheses for the corresponding dependent measures.

\section{Counterbalancing}

To rule out order and level-specific effects, a complete counterbalance of the factors was achieved. With three conditions in this within-subject experiment, the total number of sequences required to achieve a complete counterbalance was six. We had a total of two dyads in each sequence. In each study session, the dyads played three levels during which data were collected, during this time they experienced all three variant conditions as described above.

\section{Measures}

In this study, we used annotation tools (map-plus-gameworld, map-only, none) as the levels for the IV. To evaluate performance, time spent in each level was collected from the gameplay video recording, which was used to analyze how much time each pair spent to finish the tasks and number of times they used the annotations.

To assess workload, NASA-TLX [26] was chosen as it is the most commonly used and the most widely validated of the various tools available for measuring workload [25]. NASA-TLX consists of six items measuring different aspects of workload on a 100-point scale. In this study, the weighting of NASATLX was omitted to reduce the time it took to complete the questionnaire [38]. Moroney et al. argue that the use of unweighted NASA-TLX scores is valid and adequate when the time is limited [38].

The Game Experience Questionnaire [29] was employed in this study to evaluate whether the noted annotation styles would differentially impact player experience, as compared to no annotations at all. IJsselsteijn et al. [29] argue that the game experience dimensions: immersion, tension, competence, flow, negative affect, positive affect, and challenge are important elements to assess gameplay experience. After each level, we administered the in-game GEQ and follow-up questions regarding game difficulty and interface usefulness.

The in-game GEQ consisted of seven dimensions with two items per dimension. These dimensions were: positive affect, negative affect, tension, flow, challenge, and competence. In this study, 'immersion' was not included, because it was less important to the current research and also to reduce the time it took to complete the questionnaire. The 'positive' dimension assessed the perceived degree of contentment. The 'negative' dimension assessed the perceived degree of tiredness and boredom. The 'tension' dimension assessed the perceived degree of irritability and frustration. The 'flow' dimension assessed the perceived degree of absorption in the game. The 'challenge' dimension assessed the perceived effort put into the game. The 'competence' dimension assessed the perceived degree of skill and success felt in the game.

All GEQ items and follow-up questions were measured using five-point intensity scales with points anchored at 'not at all' (0), 'slightly' (1), 'moderately' (2), 'fairly' (3), and 'extremely' (4). For our analyses of GEQ, we used the mean value of the two items per dimension.

\section{Design of $\mathrm{TeCP}$}

To identify the effectiveness of annotation interfaces in distributed multiplayer games, we designed $T e C P$, a two-person cooperative game in which players' avatars are physically separated in the gameworld. The players must work together by communicating and annotating the map and by moving through and manipulating the gameworld. The game is designed to evaluate different types of annotation interfaces and their effect on players' performance and planning activities.

The design of the game was informed by our prior research on disaster response planning, in which we developed game design patterns to engage players in disaster-response-style planning activities [48]. In building on that work, we make use of the following patterns:

- Collaborative Planning ${ }^{1}$ : players should interact with space on a map to specify future activities that will be undertaken by players.

- EMERGENT OBJeCtives: objectives in the game may be discovered, developed, or lost as particular game scenarios play out. Not all objectives in the game need to be accomplished.

- Developing intelligence: players should make informed decisions about how to collect information in a gameworld, and need to make judgments of its value.

The game is also informed by design aspects of Due Process [21], Portal 2 [53], and Monument Valley [51].

\section{Game Mechanics}

Play in $\mathrm{TeCP}$ involves two players moving their avatars through the gameworld, while communicating, to complete puzzles. Specifically, the game develops the following mechanics for movement in the gameworld:

- moving the avatar in all directions, subject to gravity;

${ }^{1}$ SMALL CAPITALS are used for pattern titles in pattern languages [3]. 

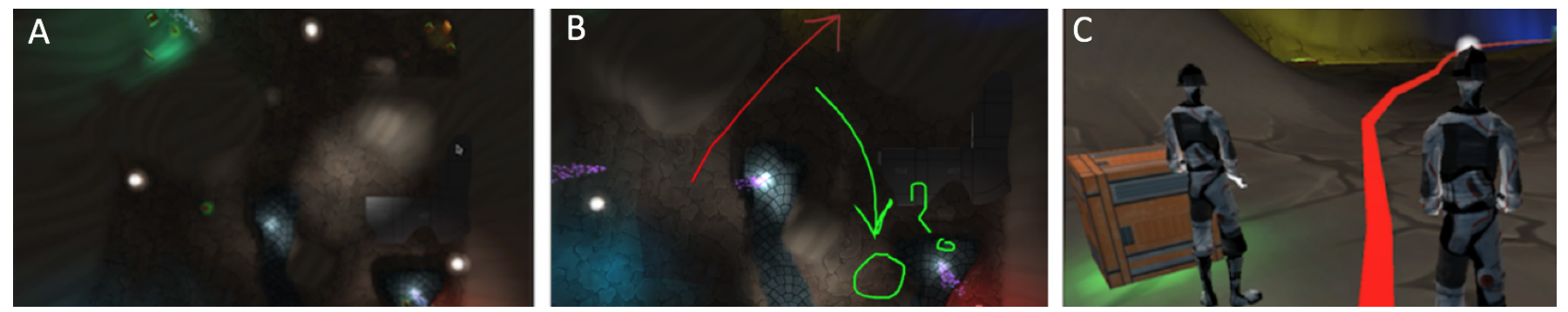

Figure 1. A: The top-down map allows players to see part of the play space to develop strategies and plans. B: Players use the mouse cursor to draw on the map to establish plans and strategies. Drawings are colored differently to identify and follow. C: The drawing is made visible directly on the gameworld, enabling its use during action gameplay, which gives the players the ability to follow their plan, and to develop it in real time.

- carrying, placing, and stacking cubes handled by the avatar to complete the different tasks;

- pressing buttons in the gameworld to open doors or activate elevators by positioning the avatar over the button objects;

- jumping on platforms that raise the character to higher platforms to collect out-of-reach objects;

- teleporting through portals to move around the gameworld; and

- switching viewpoints with the use of specific keys that toggle different game views.

In addition, the game develops a set of mechanics to support communication and planning:

- text messages can be sent to the other player in the game through the in-game text chat; and

- drawing annotations, by clicking and dragging the mouse cursor over the map interface to create freehand annotations (when not disabled in an experimental condition).

\section{Planning Interfaces and Annotations}

To engage players in COLLABORATIVE PLANNING [48], the game allows players to interact with space on a map to specify the future activities that will be undertaken. The top-down map (Figure 1, A), allows players to see part of the play space so as to plan and to develop strategies. The drawing system serves as a CCM, allowing players to collaboratively prioritize actions and assign the objectives (e.g., which path each player will take). To investigate how annotations can facilitate collaborative planning in games, we designed two alternative designs for the annotation tool: map-plus-gameworld annotation and map-only annotation. In our experiment, we compare these two designs with a control that does not allow annotations:

- Map-plus-gameworld annotation: Players can create freehand annotations on the map, which are shared between teammates. These annotations persist for 60 seconds and can be viewed in both the map and the gameworld, enabling their use during action gameplay (Figure 1, C).

- Map-only annotation: As map-plus-gameworld, but annotations do not appear in the gameworld (Figure 1, B). Players must access the map when they want to view plans.

\section{Level Design}

We designed three different game levels. Players need to complete a set of three different tasks (similar to missions or quests in video games) in each level. The level complexity is defined by the collaborative tasks, which require players to perform a sequence of steps.

\section{Time Pressure}

Although a number of games that provide players with pings and annotations are highly intensive and time-critical games, $\mathrm{TeCP}$ is not. We designed the game with only moderate time pressure, to encourage players to spend time collaborating and planning. The moderate time pressure gives players enough time to develop a strategy, collaboratively plan actions in advance, and then execute their plan. Each level lasts for 15 minutes, during which the players need to complete the three different collaborative tasks. In this game, we expect an expert player would finish the levels in 5 to 10 minutes.

\section{Communication Channels}

$\mathrm{TeCP}$ provides players with only one language-based communication channel: text chat. Text chat allows players to send text messages to the other player in the game. Players can use the text chat as a way to communicate their plans, ideas, or comments to the other player. Verbal communication is not available in the game. We excluded verbal communication so as to control how much dyads can communicate in the game. Focusing only on non-verbal communication enabled us to assess how annotations impact or complement this type of communication. Prior studies showed that text chat in time-critical games (e.g., Dota 2 [54]) can be distracting and negatively affects player performance [34], however, $\mathrm{TeCP}$ was designed with moderate time pressure, so that using text chat would not hamper performance [57]. Although chat is the only direct communication channel available, being able to draw and annotate on the map gives the players another way to exchange information.

\section{Gameplay Scenario}

At the beginning of the game, players have a set of objectives that need to be completed. For example, finding and placing three different colored cubes, which are distributed in the gameworld, in their associate place to complete the level. Players start by collaboratively establishing a plan using the top-down view of the gameworld, which details some of the objects in the game. Using the annotation interfaces, which are inspired by Due Process [21], players may collaboratively draw on the map to mark locations of cubes, draw pathways, and divide tasks to complete objectives. All levels require the players to work together to reach a common goal. Cooperative goals in the game include: pressing buttons to open doors for a 
teammate and activating an elevator to reach higher platforms. Dependences in the game force collaboration. Specific objects in the game are assigned to one of the players (e.g., cubes); to manipulate these game objects, players need to coordinate activities and divide tasks as required. Once they complete each task, the game displays a message to inform them of the completion of the task. Players have the freedom to plan and make their own decisions on which task or objective needs to be completed first. This allows players to rely on their own judgment and plan without any restrictions from the game rules.

\section{Apparatus}

Participants played the TeCP game in a laboratory with two desks facing back to back. Both desks were equipped with identical computers: an Intel Xeon CPU E31241 v3 3.50GHz processor with 16GB RAM; an NVIDIA Quadro K2200 Graphics card; a 1080p LED 27-inch, wide screen monitor; a keyboard and mouse; and an Afterglow Universal wireless headset. Because both players were co-located in the same room, and to prevent them from communicating via voice, the headset played background music and the auditory feedback from the game, which prevented them from hearing each other.

\section{Data Collection}

During the user study, gameplay video was recorded using Active Presenter. In addition to the video recordings, we collected self-reported demographics, prior gaming experience, NASA TLX, and GEQ data.

\section{Study Protocol}

After being shown into the lab, participants provided informed consent. The participants were then asked to complete a demographics and prior gaming experience questionnaire. The demographics questionnaire covered age, gender, education, and also included general questions about the subject's familiarity with collaborative games, general gaming expertise, and length of relationship with the partner. After playing each level, the NASA TLX [26] and GEQ [29] were administered, along with questions to assess the usefulness of the annotation tools.

Before playing, we ensured that participants were familiar with the game, as well as with its cooperative-play mechanics. Each pair was given a ten-minute tutorial which involved most of the game mechanics used in the game. The tutorial included a written step-by-step walk through, which directed the players on how to complete the tasks in the game.

\section{RESULTS}

Repeated-measures analyses of variance (ANOVAs) were used to assess the impact of annotation style on level completion time, NASA TLX scores, and GEQ scores.

\section{Exploratory Correlation Analyses}

Exploratory correlation analyses were conducted to determine whether level completion time correlated with our secondary dependent measures, dyad means scores across the sub groupings of the TLX and GEQ (due to multiple analyses, $\alpha$ level was set to 0.005 to control for $\alpha$ inflation). Level completion time significantly correlated with mean temporal demand $[r(35)=.46, p<.01]$ and perceived performance success

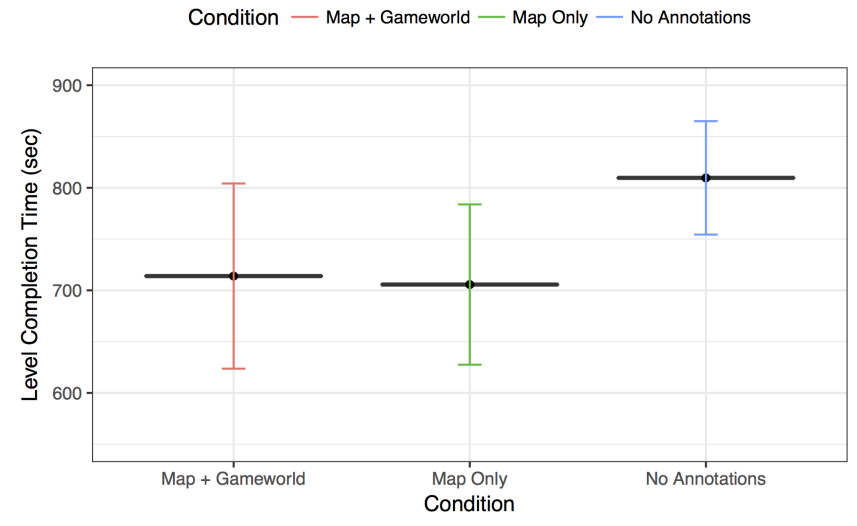

Figure 2. Average time in seconds that dyads took to complete each level; error bars represent $95 \%$ confidence intervals.

$[r(35)=.63, p<.001$; performance is reverse coded with smaller numbers indicating greater perceived success]. Other tested relationships were not statistically significant.

\section{Level Completion Time Findings}

Level completion time, an objective metric, was evaluated at the team level because this parameter did not vary between teammates (levels were completed concurrently). Players generally managed to complete the levels within the allocated time; in 4 (of 36) instances dyads exhausted their time prior to completing all of the goals. This slightly reduced the variance in our data.

Repeated-measures ANOVAs was conducted with annotation condition (map-plus-gameworld, map-only, none) as the IV and level completion time as the DV. The analysis revealed that annotation condition had a significant impact on time spent in each level $\left[F(2,11)=3.95, p<.05, \eta_{p}^{2}=.26\right]$. Pairwise comparisons revealed that participants completed levels marginally more quickly when they annotated the map-only $(p<.06)$ and the map-plus-gameworld $(p<.07)$ versus without annotations (Figure 2). Level completion times did not differ between the two annotation conditions. Thus, hypothesis H1a was supported and H1b was not.

\section{Workload Findings}

Workload, a subjective metric, was evaluated at the individual level because this parameter did vary between teammates. While it is possible to compute team versions of those metrics (e.g., by averaging the scores), there is no theoretical reason to do so and this transformation would result in a loss of meaningful variance.

Repeated-measures ANOVAs were conducted using the annotation condition (map-plus-gameworld, map-only, none) as the within-subject factor for scores along each of the TLX sub groupings: effort, frustration, performance, mental demand, physical demand, and temporal demand. Annotation type significantly impacted self-reported effort $[F(2,22)=$ $3.63, p<.05, \eta_{p}^{2}=.25$ ] and perceived performance success $\left[F(1,22)=3.78, p<.05, \eta_{p}^{2}=.26\right]$ scores, and marginally impacted frustration scores $\left[F(2,22)=3.22, p<.06, \eta_{p}^{2}=\right.$ .23]. Pairwise comparisons showed that participants perceived 


\begin{tabular}{lcc}
\multicolumn{1}{c}{ Level } & $M$ & $S D$ \\
map-plus-gameworld annotations & 33.75 & 19.65 \\
map-only annotations & 18.75 & 11.76 \\
none & N/A & N/A
\end{tabular}

Table 2. The mean number of annotations used per dyad during each condition when the annotations were available.

their performance to be more successful on the map-plusgameworld condition $(M=33.75)$ than when using no annotations ( $M=48.96$; performance is reverse-coded). Pairwise comparisons also showed that when participants annotated in the map-plus-gameworld, their frustration scores $(M=44.6)$ were marginally lower $(p<.1)$ than when not annotating $(M=63.12)$. No other pairwise comparisons were significant.

The impact of annotation type on mental demand $[F(2,22)=$ $1.48, p=.25]$, physical demand $[F(2,22)=1.56, p=.23]$, and temporal demand $[F(2,22)=2.16, p=.14]$ was not significant.

\section{Annotation Use Findings}

To test H2, we investigated the number of times the players used the annotation tool in the game. We analyzed the resulting videos to count the number of times the dyads used the annotation tool. The average number of annotations used is described in Table 2. A paired-samples t-test showed that participants annotated more in the map-plus-gameworld level $(M=33.75)$ than in the map-only $(M=18.75)[t(11)=2.46$, $p<.05]$. Thus, hypothesis $\mathbf{H} \mathbf{2}$ was supported.

\section{GEQ Findings}

We used the game experience questionnaire to assess the dimensions of game experience. Repeated-measures ANOVAs were conducted using the annotation condition (map-plusgameworld, map-only, none) as the within-subject factor for scores along each of the GEQ sub groupings. Annotation condition did not significantly impact any of the GEQ subgrouping scores: challenge $[F(2,22)=.065, p=.93]$, competence $[F(2,22)=.18, p=.83]$, flow $[F(2,22)=.34$, $p=.71]$, positive affect $[F(2,22)=1.72, p=.21]$, negative affect $[F(2,22)=2.14, p=.14]$, or tension $[F(2,22)=.78$, $p=.46]$.

\section{Game Difficulty and Interface Usefulness Findings}

Repeated-measures ANOVAs were conducted using the annotation condition (map-plus-gameworld, map-only, none) as the within-subject factor for difficulty and interface usefulness scores. While participants thought the game was somewhat easier when they annotated and that the annotation interfaces were somewhat more useful than the basic interface with no annotation, there were no statistically significant differences observed: difficulty $[F(2,22)=.49, p=.61]$ and usefulness $[F(2,22)=2.12, p=.14]$.

Additionally, we asked each participant at the end of the experiment if the annotations were useful during the game: $91.7 \%$ of responses indicated that annotations were useful and helped them during the game.

\section{Annotation Use Cases}

In the following section, we present a classification of the different use cases of the annotation tools. This is a qualitative analysis that looks at the different ways players used the annotations during the game. We found five distinct uses for the annotations: real-time way-guiding, marking locations and objects, handwriting messages, expressing emotions, and spamming. There follows a description of each use case along with supporting responses collected from participants:

Real-time way-guiding: Players used the annotation tools to guide their fellow teammate to specific locations in the game in real time (see Figure 3, A). It was observed that real-time wayguiding was an easy way for players to communicate spatial information in the game. Players follow the annotations to reach specific waypoints:

Yes [annotations were useful], specifically for the map, and way-finding aerial view. [P19]

There were cases where a more experienced player guided their teammate through the entire gameworld. Players used the annotation tools collaboratively through the top-down map to ascertain whether the other player was following the plan the team had agreed upon.

Marking locations and objects: Some objects in the game are assigned to one of the players only. To manipulate these game objects, players needed to coordinate and divide tasks according to these dependencies (Figure 3, B). Players used the top-down map to mark these objects or places and thus communicate their location to the other player:

They [the annotations] helped communicate locations and directions. [P23]

These marked locations or objects were found to be easily located by both players when annotations were used.

Handwriting messages: Players used annotations to handwrite messages on the map. In a number of cases, players wrote expressions like "here", or "is this it?" using the annotations to communicate with each other (e.g., Figure 3, C). Although the players had access to text chat, writing on the map helped them share information and mark locations.

Expressing emotions: Players used the annotations not only to plan and communicate future actions and information, but also as a way to express emotions. We observed a number of cases where players used the annotation tool to draw happy or sad emoticons to express their feelings (e.g., Figure 3, D).

Spamming: In MOBA games, ping spamming is a common issue (e.g., League of Legends [40], Dota 2 [54]), in which players send multiple pings in a short interval [34], however, we did not expect this would be the case with annotations. (there follows a snippet from the game text chat, in which one teammate is impacted negatively by the annotations):

I draw something. [P22] $\longrightarrow$ Stop drawing. [P21]

We observed a number of cases where one teammate used annotations excessively to irritate their teammate or to attract their attention, annotation spamming (Figure 3, E). 

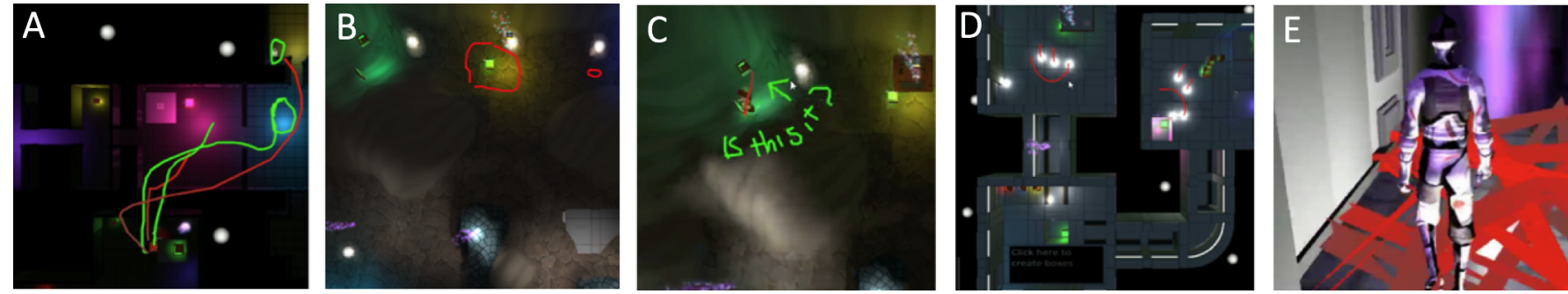

Figure 3. Examples of the use cases of the annotations. A: Real-time way-guiding in the gameworld and map. B: Using annotations to mark locations and objects on the map. C: Player's handwriting message on the map. D: Using annotation to express emotions. E: Using annotations for spamming.

\section{Player Experience Findings}

We examined the participants' responses to the annotation interfaces they used during the game. In the following discussion, we present reflections and quotes from the questionnaires on how the participants perceived the usefulness of these interfaces, as well as how they affected their play experience and communication skills.

\section{Improve Communication}

Players used the annotations to complement their communication efforts and help establish a new communication channel:

Yes it [the annotation tool] was really helpful to talk to each other. [P1]

Players stated that the absence of annotations had a significant impact on their ability to communicate with each other:

It [the annotations] was useful and when it was not there anymore I felt like we lost a communication skill. [P4]

For example, when there was a language barrier between team members, annotations helped them to communicate and overcome this communication overhead ${ }^{2}$ :

\section{It [the annotation tool] was extremely useful especially because my partner had problems to communicate in English. [P9]}

Ease of communication between the players was observed when using annotations in the game:

Yes it [the annotation tool] was very helpful to show the other person what you want him to do. Also to show directions or buttons/elevators. I missed it in the last level and it was way harder to describe. [P5]

However, when annotations were absent from the game, players' perception of difficulty or challenge changed:

I was very confused when there was no drawing [annotation]. [P12]

Using annotations supported players in improving teamwork, because they could communicate through gameplay:

Oftentimes pictures spoke louder than words and we pointed out what we meant by drawing [annotating]. Because when we were describing with words we described it relative to us. [P17]

\footnotetext{
${ }^{2}$ Communication overhead is the cost in time, bandwidth, cognition, etc. of communicating [35].
}

\section{Waypoint Support}

Annotations helped players to create waypoints to display on the map, which enabled them to collaboratively plan a sequence of points that they could follow:

Yes it [the annotation tool] did help. Allowed us to map out where each box was and draw a path for the other player to help guide them where things/boxes are. [P8]

These waypoints could be used to guide players to different locations in the game, and were more beneficial in complex game levels:

[The annotation] helped indicate what elevator was moving or where you wanted to go to the other player. It would be even more useful in more complex or convoluted levels. [P14]

\section{Gameworld Annotations:}

The study results show that the usage of annotations increased when they were visible in the gameworld. Players said that annotations visible in the gameworld were useful for planning:

The drawing that persisted [visible in the gameworld] was very helpful in identifying destinations and charting a course which I could follow. When the drawing did not persist [visible only on the map], it was mainly helpful in communicating objectives between players. [P16]

When these annotations were visible in the gameworld, they were perceived by players as being most helpful:

It [annotation] was helpful especially because you could see it in-game not only on the minimap. [P6]

\section{Level of Expertise:}

Expert players preferred not to use annotations during the game, these players had already developed communication modalities and mostly relied on traditional ways to communicate:

Due to being used to using traditional chat, the drawing [annotations] didn't play as much of a role as I thought it would. Simply typing through the chat felt sufficient and I eventually forgot about drawing. [P13]

This response was collected from a participant that indicated in the gaming experience survey that he plays games every day, for more than 3 hours. 


\section{DISCUSSION AND DESIGN IMPLICATIONS}

In this section, we draw out the key themes in our research, connecting together findings from our experiments, connecting the use cases into our discussion. According to our results, most dyads used these annotations to help them establish a shared plan and enhance their ability to work as a team, which reduced frustration, sped up communication, and enhanced perceived and actual performance. The main goal of this study was to investigate how different annotation tools facilitate collaborative planning in games; we found that they support collaborative planning through enhancing communication and removing impediments to coordination, and that there is value in annotating the gameworld rather than just the map interface. We also noted how annotation use differs by expertise, and point to ways in which this might balance play. Furthermore, we developed five use cases (real-time way-guiding, marking locations and objects, handwriting messages, expressing emotions, and spamming), which can be grouped into two larger categories:

1. supporting collaborative planning; and

2. minimizing hindrances and offloading work.

\section{Supporting Collaborative Planning}

The results suggest that annotations are a great tool to establish a shared understanding of game objectives, plan and divide tasks among players, and improve team coordination. While annotations have been used in games, they are not designed to help players collaboratively plan in advance, but, rather react and call attention to emergent play states through marking locations and objects. Our study revealed that, to help players engage in collaborative planning, games need to supply them with tools and interfaces that help build a shared understanding of the task at hand, enabling the use cases of real-time wayguiding and handwriting messages. Annotations in games help players develop a shared mental model of the gameworld and provide them with a set of tools that enable coordination and teamwork, in service to both planning and reaction. Through the use cases of handwriting messages, expressing emotions, and (even) spamming, we see that players used the annotations not only to plan actions in advance, but as a communication tool and, thus, a skill that can be fostered. While alternative affordances in map annotation interfaces may develop new use cases (e.g., providing the ability to write text would likely obviate handwriting messages), we see that annotations are valuable to players: such interfaces can help them improve communication skills that lead to better teamwork and reduce communication barriers.

Though annotations proved to be a great tool to establish collaborative planning and reduce communication overhead, players do not often use it in time-critical games, so our use cases do not appear. We believe the reason for this is that players do not have enough time in time-critical games (e.g., Dota 2) to create freehand annotations, which require more time compared to pings. Thus players rely mostly on low-cost pings when there are time constraints. [34,59].

Mutual understanding of the objectives between the players are essential to their success in distributed multiplayer games. We believe that annotations help players to establish a common ground [12], which enables them to easily collaborate with each other and reduces the need to communicate. Because verbal communication is not always available, people rely on other means of communication. Having the ability to collaboratively establish plans through annotating the gameworld helps improve situation awareness and helps players gain a shared understanding of the game objectives [28].

\section{Minimizing Hindrances and Offloading Work}

One potential benefit of providing annotations in games is to reduce cognitive effort and workload caused by remembering plans and communication overhead. Although we did not observe significant differences in workload in our study due to the use of annotation, trends were in the right direction and we found that annotations significantly lessened frustration. The use of real-time way-guiding by participants is one means of offloading (cognitive) work, which is not possible without a gameworld visualization. This benefit of visual information has been researched in several studies [16, 17, 20,33] showing that pairs perform better when they are using video tools that provide views of the workspace than when they are using audio or text-based communication alone. Based on our observations, players' perception of the level of difficulty in the game changes according to the availability of the annotation tools. Our findings point to real-time visible annotations being a preferred mode of planning game moves. Without annotations, players need to keep track of their plans and next moves in the game. Players need to synchronize their activities and maintain a shared situation awareness of the game.

To aid players, annotations can help to plan and divide tasks collaboratively and keep track of the developed plan. Annotations can be created on the minimap, and by clicking and dragging the mouse in this area, more information can be expressed about the players' strategic plan than otherwise. However, having the annotation tool visible in the gameworld provides players with a variety of options for awareness, easy targeting, and concise communication and guiding; these are important when players need to perform and follow a strategy to undertake tasks in a game.

\section{Value of Annotating the Gameworld}

Not surprisingly, our results show that when annotations are visible in both the gameworld and map interface, their usage significantly increases. These results are in line with prior research that suggested to increase annotations use in games, "annotations might be used more often if they were more salient and more easily created. For example, annotations could be created and viewed directly on the gamespace rather than the minimap." [59, p. 1981]. Providing players with annotation tools and options has the potential to improve how players collaboratively plan in games and virtual workspaces. Making these tools customizable by players, in which they can enable/disable them or choose different ways they are presented in the gameworld have the potential to increase their usage and reduce the issues caused by annotation spamming.

While trends were similar in the map-only condition, significant improvements in perceived performance and reduced frustation were only observed in the map-plus-gameworld annotation condition. When annotations are visible in the 
gameworld, they are no longer only used to collaboratively plan and communicate locations, they transform the formulated plan from a "paper" model to sequence of situated actions and provide an understanding of how physical actions and language are integrated to perform joint tasks and ground communication [19].

\section{Novices and Experts}

Expert players have a tendency to not use annotations during the game. We found that expert players had already built strong communication and planning skills from spending hours of playing games. These players are familiar with using voice, text, and CCMs, which are far more common than annotations. We expect that this difference comes down to prior experience with the present state of recent games, but more research is needed. Visual annotations could be used to complement this well-established expertise communication between players, yet it is presently not a part of their repertoire. Currently, annotations are not designed in games to help both expert and novice players. Expert players tend not to use these tools, because of their prior game experience.

Our results align with prior research on the use of annotations in helping remote collaborators to work together. Fussell et al. [17] investigated the use of annotations over video streams to support remote help-giving or instruction. Local workers and remote helpers interact with each other by allowing helpers to overlay pen-based gestures, or annotations, onto a video stream of the worker's task space. Minneman and Bly [37] found that collaborative drawing tools help both two- and three-member teams to collaborate effectively. Annotations in games can open up opportunities for novices who would not otherwise be able to play with friends that have a higher expertise [30]. This means that annotations could act as a form of player balancing $[6,10,55]$ and offer opportunities for designers to use them to narrow the gap in skills between novice and expert players. Enabling collaborative play in distributed multiplayer games through annotation tools enables players to feel more competent in their game collaborations, to perform better, and to experience less frustration because of communication barriers.

\section{LIMITATIONS}

We did not collect data on the long-term effects or value of the impact of annotations on team performance and collaborative planning. We suggest that valuable future work in this space could investigate the long-term value of annotations in virtual training scenarios, such as disaster response training and serious games. Verbal communication in distributed multiplayer games is used commonly, however, we did not look at the impact of combining voice communication and annotations on player performance and collaborative planning. To do this, we would have to add one or two more conditions to the experiment, which requires a higher number of participants and was beyond the scope of this work. However, future work could investigate the use of different types of annotations (e.g., annotating map with text or markers) and combining them with text chat and verbal communication.

\section{FUTURE WORK}

This work helps us understand how collaborative planning in games can be supported using different mechanics and interfaces. We see an opportunity to develop games and interfaces that help players engage in COLLABORATIVE PLANNING for serious games and game design in general. Future work should address how annotations in collaborative training simulations improve players planning and sensemaking skills in different game environments such as virtual and mixed reality $[8,45]$. For game design, a future direction could be to investigate how different CCMs, including pings, maps, and annotations affect player performance and how they support collaborative planning in games. Future work will also look at applications for annotations: disaster response, combat, e-sports training, and team-training, in general.

\section{CONCLUSION}

Our study continues prior research that investigated the impact of CCMs on player performance in collaborative games $[34,49,52,59]$. We focused on the effect of using annotations on player performance, workload, and experience in distributed multiplayer games and how they engage players in collaborative planning. We found that annotation tools improved actual and perceived performance, reduced frustation, and enhanced communication. Moreover, the use of annotations increased when these annotations are visible in the gameworld and map when compared to only being visible on the map. Furthermore, we identified five different use cases for the annotations: real-time way-guiding, marking locations and objects, handwriting messages, expressing emotions, and spamming.

Based on these findings, we see an opportunity to design games that focus on engaging players in collaborative planning tasks. Using annotations in games opens up further research on how different gestures and non-verbal communication can be used to facilitate remote planning and collaborations. This study helps us define potential advantages of annotation interfaces and their implications for the design of collaborative games. These annotation tools could have further benefits beyond games; for example in virtual training scenarios such as disaster response training.

\section{ACKNOWLEDGMENTS}

We would like to thank the other students in Digital Game Design for their feedback on early versions of $T e C P$. This material is based upon work supported by the National Science Foundation under Grant Nos. IIS-1651532 and IIS-1619273. We also acknowledge support from the Social Sciences and Humanities Research Council of Canada 895-2011-1014, the Natural Sciences and Engineering Research Council of Canada RGPIN-418622-2012, the Canada Foundation for Innovation 35819, and Mitacs IT07255.

\section{REFERENCES}

1. Ernest Adams and Joris Dormans. 2012. Game Mechanics: Advanced Game Design (1st ed.). New Riders Publishing, Thousand Oaks, CA, USA.

2. David S. Alberts, John J. Garstka, Richard E. Hayes, and David A. Signori. 2001. Understanding information age warfare. Technical Report. DTIC Document. 
3. Christopher Alexander, Sara Ishikawa, Murray Silverstein, Max Jacobson, Ingrid Fiksdahl-King, and Shlomo Angel. 1977. A Pattern Language: Towns, Buildings, Construction. Center for Environmental Structure, Vol. 2. Oxford University Press, New York, NY, USA.

4. Sultan A. Alharthi, Ruth Torres, Ahmed Khalaf, and Zachary O. Toups. 2017. The Maze: Enabling Collaborative Planning in Games Through Annotation Interfaces. In Extended Abstracts Publication of the Annual Symposium on Computer-Human Interaction in Play (CHI PLAY'17 Extended Abstracts). ACM, New York, NY, USA, 615-620. DOI : http://dx.doi.org/10.1145/3130859.3130864

5. Chris Baber, James Cross, Paul Smith, and Dengel Robinson. 2007. Supporting Implicit Coordination Between Distributed Teams in Disaster Management. Springer Berlin Heidelberg, Berlin, Heidelberg, 39-50. DOI : http://dx.doi.org/10.1007/978-3-540-75668-2_5

6. Scott Bateman, Regan L. Mandryk, Tadeusz Stach, and Carl Gutwin. 2011. Target Assistance for Subtly Balancing Competitive Play. In Proceedings of the SIGCHI Conference on Human Factors in Computing Systems (CHI '11). ACM, New York, NY, USA, 2355-2364. DOI :

http://dx.doi.org/10.1145/1978942 . 1979287

7. Staffan Björk, Sus Lundgren, and Jussi Holopainen. 2003. Game Design Patterns. In Level Up - Proceedings of Digital Games Research Conference 2003.

8. Elizabeth M. Bonsignore, Derek L. Hansen, Zachary O. Toups, Lennart E. Nacke, Anastasia Salter, and Wayne Lutters. 2012. Mixed Reality Games. In Proceedings of the ACM 2012 Conference on Computer Supported Cooperative Work Companion (CSCW'12). ACM, New York, NY, USA, 7-8. DOI : http://dx.doi.org/10.1145/2141512.2141517

9. Barry Brown and Marek Bell. 2004. CSCW at Play: 'There' As a Collaborative Virtual Environment. In Proceedings of the 2004 ACM Conference on Computer Supported Cooperative Work (CSCW '04). ACM, New York, NY, USA, 350-359. DOI : http://dx.doi.org/10.1145/1031607.1031666

10. Jared E. Cechanowicz, Carl Gutwin, Scott Bateman, Regan Mandryk, and Ian Stavness. 2014. Improving Player Balancing in Racing Games. In Proceedings of the First ACM SIGCHI Annual Symposium on Computer-human Interaction in Play (CHI PLAY '14). ACM, New York, NY, USA, 47-56. DOI: http://dx.doi.org/10.1145/2658537.2658701

11. Victor Cheung, Y.-L. Betty Chang, and Stacey D. Scott. 2012. Communication Channels and Awareness Cues in Collocated Collaborative Time-critical Gaming. In Proceedings of the ACM 2012 Conference on Computer Supported Cooperative Work (CSCW'12). ACM, New York, NY, USA, 569-578. DOI : http://dx.doi.org/10.1145/2145204.2145291
12. Herbert H. Clark. 1996. Using Language. Cambridge University Press.

13. Mica R. Endsley. 1995. Toward a theory of situation awareness in dynamic systems. Human Factors 37, 1 (1995), 32-64.

14. Mica R. Endsley. 2000. Theoretical Underpinnings of Situation Awareness: A Critical Review. In Situation Awareness Analysis and Measurement, Mica R. Endsley and D J Garland (Eds.). Lawrence Erlbaum Associates, Mahwah, NJ, USA, 3-6.

15. Omid Fakourfar, Kevin Ta, Richard Tang, Scott Bateman, and Anthony Tang. 2016. Stabilized Annotations for Mobile Remote Assistance. In Proceedings of the 2016 CHI Conference on Human Factors in Computing Systems (CHI'16). ACM, New York, NY, USA, 1548-1560. DOI : http://dx.doi.org/10.1145/2858036.2858171

16. Susan R. Fussell, Leslie D. Setlock, and Elizabeth M. Parker. 2003. Where do helpers look?: gaze targets during collaborative physical tasks. In CHI'O3 Extended Abstracts on Human Factors in Computing Systems. ACM, 768-769.

17. Susan R. Fussell, Leslie D. Setlock, Jie Yang, Jiazhi Ou, Elizabeth Mauer, and Adam D. I. Kramer. 2004. Gestures over Video Streams to Support Remote Collaboration on Physical Tasks. Hum.-Comput. Interact. 19, 3 (Sept. 2004), 273-309. DOI : http://dx.doi.org/10.1207/s15327051hci1903_3

18. Dedre Gentner and Albert L. Stevens. 1983. Mental Models. Lawrence Earlbaum Associates, Hillsdale, NJ, USA.

19. Darren Gergle, Robert E. Kraut, and Susan R. Fussell. 2004a. Action as language in a shared visual space. In Proceedings of the 2004 ACM conference on Computer supported cooperative work. ACM, 487-496.

20. Darren Gergle, David R. Millen, Robert E. Kraut, and Susan R. Fussell. 2004b. Persistence matters: Making the most of chat in tightly-coupled work. In Proceedings of the SIGCHI conference on Human factors in computing systems. ACM, 431-438.

21. Giant Enemy Crab. 2015. Due Process. Game [Windows]. (March 2015). Giant Enemy Crab, Seattle, WA, USA.

22. Carl Gutwin and Saul Greenberg. 2002. A Descriptive Framework of Workspace Awareness for Real-Time Groupware. Computer Supported Cooperative Work (CSCW) 11, 3 (Sep 2002), 411-446. DOI: http://dx.doi.org/10.1023/A: 1021271517844

23. Carl Gutwin, Saul Greenberg, and Mark Roseman. 1996. Workspace Awareness in Real-Time Distributed Groupware: Framework, Widgets, and Evaluation. Springer London, London, 281-298. DOI : http://dx.doi.org/10.1007/978-1-4471-3588-3_18 
24. William A. Hamilton, Oliver Garretson, and Andruid Kerne. 2014. Streaming on Twitch: Fostering Participatory Communities of Play Within Live Mixed Media. In Proceedings of the SIGCHI Conference on Human Factors in Computing Systems (CHI'14). ACM, New York, NY, USA, 1315-1324. D0I : http://dx.doi.org/10.1145/2556288.2557048

25. Sandra G. Hart. 2006. Nasa-Task Load Index (NASA-TLX); 20 Years Later. Proceedings of the Human Factors and Ergonomics Society Annual Meeting 50, 9 (2006), 904-908. D0I : http://dx.doi.org/10.1177/154193120605000909

26. Sandra G. Hart and Lowell E. Staveland. 1988. Development of NASA-TLX (Task Load Index): Results of Empirical and Theoretical Research. Advances in Psychology 52 (1988), 139 - 183. DOI : http://dx.doi .org/10.1016/S0166-4115(08)62386-9 Human Mental Workload.

27. Christian Heath and Paul Luff. 2000. Team work: Collaboration and control in London Underground line control rooms. In Technology in Action, Roy Pea, John Seely Brown, and Christian Heath (Eds.). Cambridge University Press, Cambridge, UK, 88-124.

28. David Huffaker, Jing Wang, Jeffrey Treem, Muhammad A. Ahmad, Lindsay Fullerton, Dmitri Williams, Marshall Scott Poole, and Noshir Contractor. 2009. The social behaviors of experts in massive multiplayer online role-playing games. In Computational science and engineering, 2009. CSE'09. International conference on, Vol. 4. IEEE, 326-331.

29. Wijnand IJsselsteijn, YAW De Kort, and Karolien Poels. 2008. The game experience questionnaire. Manuscript in preparation (2008).

30. Colby Johanson, Carl Gutwin, and Regan L. Mandryk. 2017. The Effects of Navigation Assistance on Spatial Learning and Performance in a 3D Game. In Proceedings of the Annual Symposium on Computer-Human Interaction in Play (CHI PLAY'17). ACM, New York, NY, USA, 341-353. DOI :

http://dx.doi.org/10.1145/3116595.3116602

31. David H. Jonassen and Philip Henning. 1996. Mental models: Knowledge in the head and knowledge in the world. In ICLS '96: Proceedings of the 1996 International Conference on Learning Sciences. International Society of the Learning Sciences, 433-438.

32. Kristine Jørgensen. 2013. Gameworld Interfaces. MIT Press, Cambridge, MA, USA.

33. Robert E. Kraut, Darren Gergle, and Susan R. Fussell. 2002. The use of visual information in shared visual spaces: Informing the development of virtual co-presence. In Proceedings of the 2002 ACM conference on Computer supported cooperative work. ACM, 31-40.

34. Alex Leavitt, Brian C. Keegan, and Joshua Clark. 2016. Ping to Win?: Non-Verbal Communication and Team Performance in Competitive Online Multiplayer Games.
In Proceedings of the 2016 CHI Conference on Human Factors in Computing Systems (CHI'16). ACM, New York, NY, USA, 4337-4350. DOI :

http://dx.doi.org/10.1145/2858036.2858132

35. Jean MacMillan, Elliot E. Entin, and Daniel Serfaty. 2004. Communication Overhead: The Hidden Cost of Team Cognition. In Team Cognition: Understanding the Factors that Drive Process and Performance, Eduardo Salas and Stephen M. Fiore (Eds.). American Psychological Association, Washington, DC, USA, 61-82.

36. John E. Mathieu, Tonia S. Heffner, Gerald F. Goodwin, Eduardo Salas, and Janis A. Cannon-Bowers. 2000. The influence of shared mental models on team process and performance. (2000). DOI :

http://dx.doi.org/10.1037/0021-9010.85.2.273

37. Scott L. Minneman and Sara A. Bly. 1991. Managing a Trois: A Study of a Multi-user Drawing Tool in Distributed Design Work. In Proceedings of the SIGCHI Conference on Human Factors in Computing Systems (CHI '91). ACM, New York, NY, USA, 217-224. DOI : http://dx.doi.org/10.1145/108844.108893

38. William F. Moroney, David W. Biers, F. Thomas Eggemeier, and Jennifer A. Mitchell. 1992. A comparison of two scoring procedures with the NASA Task Load Index in a simulated flight task. In Proceedings of the IEEE 1992 National Aerospace and Electronics Conference (NAECON 1992). IEEE, 734-740. DOI : http://dx.doi .org/10.1109/NAECON.1992.220513

39. Michael Riegler, Mathias Lux, Vincent Charvillat, Axel Carlier, Raynor Vliegendhart, and Martha Larson. 2014. VideoJot: A Multifunctional Video Annotation Tool. In Proceedings of International Conference on Multimedia Retrieval (ICMR '14). ACM, New York, NY, USA, 534:534-534:537. DOI : http://dx.doi.org/10.1145/2578726.2582621

40. Riot Games. 2009. League of Legends. Game [Windows]. (October 2009). Riot Games, Los Angeles, California, USA.

41. Bryan C. Russell, Antonio Torralba, Kevin P. Murphy, and William T. Freeman. 2008. LabelMe: A Database and Web-Based Tool for Image Annotation. International Journal of Computer Vision 77, 1 (May 2008), 157-173. DOI : http://dx.doi . org/10.1007/s11263-007-0090-8

42. Eduardo Salas, Terry L. Dickinson, Sharolyn A. Converse, and Scott I. Tannenbaum. 1992. Toward an understanding of team performance and training. In Teams: Their Training and Performance, Robert W Swezey and Eduardo Salas (Eds.). Ablex Publishing Corporation, Norwood, NJ, USA, 3-29.

43. Eduardo Salas, Carolyn Prince, David P. Baker, and Lisa Shrestha. 1995. Situation Awareness in Team Performance: Implications for Measurement and Training. Human Factors 37, 1 (1995), 123-136. DOI : http://dx.doi.org/10.1518/001872095779049525 
44. Katie Salen and Eric Zimmerman. 2004. Rules of Play: Game Design Fundamentals. MIT Press, Cambridge, MA, USA.

45. Hitesh Nidhi Sharma, Sultan A. Alharthi, Igor Dolgov, and Zachary O. Toups. 2017. A Framework Supporting Selecting Space to Make Place in Spatial Mixed Reality Play. In Proceedings of the Annual Symposium on Computer-Human Interaction in Play (CHI PLAY'17). ACM, New York, NY, USA, 83-100. DOI : http://dx.doi.org/10.1145/3116595.3116612

46. Lucy A. Suchman. 1987. Plans and Situated Actions: The Problem of Human-machine Communication. Cambridge University Press, New York, NY, USA.

47. Anthony Tang, Jonathan Massey, Nelson Wong, Derek Reilly, and W. Keith Edwards. 2012. Verbal Coordination in First Person Shooter Games. In Proceedings of the ACM 2012 Conference on Computer Supported Cooperative Work (CSCW'12). ACM, New York, NY, USA, 579-582. DOI : http://dx.doi.org/10.1145/2145204.2145292

48. Zachary O. Toups, William A. Hamilton, and Sultan A. Alharthi. 2016. Playing at Planning: Game Design Patterns from Disaster Response Practice. In Proceedings of the 2016 Annual Symposium on Computer-Human Interaction in Play (CHI PLAY'16). ACM, New York, NY, USA, 362-375. DOI :

http://dx.doi.org/10.1145/2967934.2968089

49. Zachary O. Toups, Jessica Hammer, William A. Hamilton, Ahmad Jarrah, William Graves, and Oliver Garretson. 2014. A Framework for Cooperative Communication Game Mechanics from Grounded Theory. In Proceedings of the First ACM SIGCHI Annual Symposium on Computer-human Interaction in Play (CHI PLAY '14). ACM, New York, NY, USA, 257-266. D0I : http://dx.doi.org/10.1145/2658537.2658681

50. Zachary O. Toups and Andruid Kerne. 2007. Implicit Coordination in Firefighting Practice: Design Implications for Teaching Fire Emergency Responders. In Proceedings of the SIGCHI Conference on Human Factors in Computing Systems (CHI '07). ACM, New York, NY, USA, 707-716. DOI : http://dx.doi.org/10.1145/1240624.1240734

51. Ustwo Games. 2014. Monument Valley. Game [Windows]. (April 2014). Ustwo Games, London, United Kingdom.
52. Deepika Vaddi, Zachary O. Toups, Igor Dolgov, Rina Wehbe, and Lennart Nacke. 2016. Investigating the Impact of Cooperative Communication Mechanics on Player Performance in Portal 2. In Proceedings of the 42Nd Graphics Interface Conference (GI'16). Canadian Human-Computer Communications Society, School of Computer Science, University of Waterloo, Waterloo, Ontario, Canada, 41-48. DOI: http://dx.doi.org/10.20380/GI2016.06

53. Valve Corporation. 2011. Portal 2. Game [Windows]. (April 2011). Valve Corporation, Bellevue, WA, USA.

54. Valve Corporation. 2013. Dota 2. Game [Windows]. (July 2013). Valve Corporation, Bellevue, WA, USA.

55. Rodrigo Vicencio-Moreira, Regan L. Mandryk, and Carl Gutwin. 2015. Now You Can Compete With Anyone: Balancing Players of Different Skill Levels in a First-Person Shooter Game. In Proceedings of the 33rd Annual ACM Conference on Human Factors in Computing Systems (CHI '15). ACM, New York, NY, USA, 2255-2264. DOI :

http://dx.doi.org/10.1145/2702123.2702242

56. Katherine Vogt, Lauren Bradel, Christopher Andrews, Chris North, Alex Endert, and Duke Hutchings. 2011. Co-located Collaborative Sensemaking on a Large High-resolution Display with Multiple Input Devices. In Proceedings of the 13th IFIP TC 13 International Conference on Human-computer Interaction - Volume Part II (INTERACT'11). Springer-Verlag, Berlin, Heidelberg, 589-604.

http://dl.acm.org/citation. cfm?id=2042118. 2042175

57. Greg Wadley, Marcus Carter, and Martin Gibbs. 2015. Voice in Virtual Worlds: The Design, Use, and Influence of Voice Chat in Online Play. Human-Computer Interaction 30, 3-4 (2015), 336-365. DOI : http://dx.doi.org/10.1080/07370024.2014.987346

58. Karl E. Weick. 1995. Sensemaking in Organizations. Foundations for Organizational Science, Vol. 3. SAGE Publications.

59. Jason Wuertz, Scott Bateman, and Anthony Tang. 2017. Why Players Use Pings and Annotations in Dota 2. In Proceedings of the 2017 CHI Conference on Human Factors in Computing Systems (CHI'17). ACM, New York, NY, USA, 1978-2018. DOI :

http://dx.doi.org/10.1145/3025453.3025967 